\title{
Mesotelioma maligno: Experiencia clínico-patológica de 247 casos
}

\author{
RAÚL BARRERA R.*, JORGE CHAVARRÍA G.** y JORGE MORALES F.***
}

Malignant mesothelioma: Clinical and pathological features from 247 cases

Objective: This study describes the frequency, occupational, clinical, and pathological features in a large cohort of cases of Malignant Mesothelioma (MM) from the National Institute of Respiratory Diseases (INER), in Mexico. Methods: A retrospective and transversal study was carried out in medical records of patients diagnosed with MM between the years 1991 to 2007. Results: Of the 247 patients, 184 were male and 63 were female with an average age of 51-60 years. Dyspnoea and chest pain were the presenting symptoms in most of the patients. Exposure to asbestos was referred only in $34 \%$ of cases but direct exposition only was documented in five of them. Clinical features of MM patients were similar in asbestos related and non-asbestos related malignant mesothelioma. Conclusion: Although a growing tendency is observed in the number of cases with $M M$, in many of them was not possible to establish the source of asbestos exposure.

Key words: Mesothelioma; prevention \& control; asbestos; chrysotile; amphiboles.

\section{Resumen}

Objetivo: Este estudio describe la frecuencia, características patológicas, ocupación y exposición a asbesto en una cohorte grande de casos de mesotelioma maligno (MM) admitidos en el Instituto Nacional de Enfermedades Respiratorias (INER), en México. Método: Se llevó a cabo un estudio retrospectivo y transversal en los registros médicos de pacientes diagnosticados con MM entre los años 1991-2007. Resultados: De los 247 pacientes, 184 fueron hombres y 63 fueron mujeres con un promedio de edad de 51 y 60 años. Disnea y dolor torácico fueron los principales sintomas observados. Exposición a fibras de asbesto fue referida sólo en 34\% de los casos, aunque una exposición directa sólo puedo ser documentada en cinco de ellos. Las características clínicas de los pacientes con MM fueron similares en aquellos con y sin exposición a asbesto reconocida. Conclusión: Aunque se observa una tendencia creciente en el número de casos con MM, en muchos de ellos no fue posible establecer una fuente de exposición a asbesto como la causa de contaminación para el desarrollo de la enfermedad.

Palabras clave: Mesotelioma maligno; prevención y control; asbesto; crisotilo; anfiboles.

\section{Introducción}

El mesotelioma maligno (MM) es una neoplasia altamente agresiva originaria de las células serosas de la cavidad pleural y peritoneal así como el pericardio y la túnica vaginalis ${ }^{1}$. Presenta un modelo de crecimiento particular, extendiéndose a lo largo de la superficie de la pleura, invadiendo tanto la visceral como la parietal con expansión a los pulmones y tejidos adyacentes. Este tipo de tumores son de mal pronóstico y tienen un período de latencia de aproximadamente 32 años ${ }^{2}$.

En la etiología del MM, en el $70 \%$ de los casos se puede establecer con una historia o antecedente de exposición directa o indirecta a fibras de asbesto, principalmente del tipo anfiboles ${ }^{3-5}$; sin

Instituto Nacional de Enfermedades Respiratorias, México, DF, México:

* Departamento de Bioquímica y Medicina Ambiental.

** Jefe de hospitalización.

***Jefe del Servicio Clínico No. 3 y Clínica de Cáncer de Pulmón. 
embargo, no se ha podido correlacionar de manera directa la intensidad y duración de la exposición con la aparición de enfermedad pleural. Se calcula que el riesgo de desarrollar enfermedad pleural secundaria a dicha exposición es de 8\%$13 \%{ }^{6-8}$. La exposición a asbesto se asocia también con la aparición de bronquitis crónica, fibrosis pulmonar y cáncer broncogénico.

La incidencia del MM en el ámbito mundial es variable. En Estados Unidos de América (EE.UU.) el número de muertes por MM llegó a un máximo de 3.060 casos en el año 2002, después del cual se ha observado un descenso significativo ${ }^{9-11}$. Por el contrario, en el Reino Unido (UK)esta neoplasia es la responsable de 2.700 a 3.000 muertes anuales, y se calcula que estas cifras continuarán aumentando hasta un máximo de 90.000 muertes en el año 2050, después de lo cual se predice una disminución de la incidencia, como resultado de la aplicación de medidas reguladoras en la exposición y el uso de asbesto ${ }^{12,13}$. Los índices de mortalidad (IM) según la base de datos de la OMS indican que UK tiene la incidencia más alta (31,1 muertes/millón/año), pero en países como Croacia (IM = 8,8), Japón (4,8 (IM = 4,8) Argentina $(\mathrm{IM}=2,5)$, Hungría (IM $=2,5)$ y Brasil (IM $=0,5)$ se observa una tendencia significativa de incremento $^{14}$.

En México es difícil conocer la incidencia real de MM dado que existe un subregistro de este tipo de neoplasias. Aguilar y cols ${ }^{15}$, refieren que en el Seguro Social de México durante un período de 22 años (1979 a 2000) se registraron 793 muertes por $\mathrm{MM}$, siendo la mayor proporción de hombres (491 casos), respecto a las mujeres (302 casos) y con una edad promedio de 60,7 \pm 16 años. Gavira y cols, reportaron 52 casos que fueron compilados a partir de los registros clínicos de diferentes instituciones hospitalarias de México durante los años 1980-1985. En esta serie la proporción mayoritaria fue en hombres $(2,5: 1)$ y la edad promedio fue menor de 50 años ${ }^{16}$. En un estudio realizado por nuestro grupo, describimos 47 casos de MM, en los que la proporción respecto al género fue de 2,7:1, mientras que la distribución de edad fue principalmente entre los 51 y 60 años para ambos géneros ${ }^{17}$. Llama la atención que en todos estos reportes, la historia clínica sólo pudo reconocer un porcentaje bajo $(<20 \%)$ de los casos como enfermedad laboral.

\section{Objetivo}

Este estudio describe la frecuencia, tipo de ocupación, y características clínico patológicas en una cohorte grande de casos de mesotelioma maligno (MM) admitidos en el Instituto Nacional de Enfermedades Respiratorias (INER) de México.

\section{Material y Método}

Se llevó a cabo un estudio transversal, retrospectivo de 247 casos de MM diagnosticados en el INER dentro del período 1991 a 2007. La clasificación de los MM se llevó a cabo mediante las guías del International Mesothelioma Interest Group (IMIG; http://www.imig.org). En la hoja de recolección de datos, se incluyeron las siguientes variables: edad, sexo, ocupación laboral, tiempo de evolución de los síntomas (meses), exposición al asbesto, humo y otros tóxicos. Los datos clínicos considerados fueron: dolor torácico, tos, hemoptisis, pérdida de peso y disnea. Las alteraciones radiológicas se agruparon en: engrosamiento pleural, derrame pleural, retracción y desplazamiento mediastínico. Se analizaron, asimismo, los métodos diagnósticos requeridos en cada caso, tomando la información registrada del examen citológico en líquido pleural y de la biopsia pleural cerrada u obtenida por toracoscopía. En la revisión de las tomografías computadas de tórax, se valoraron las alteraciones del parénquima pulmonar, así como la presencia de adenopatías mediastínicas, masas y engrosamiento pleural. Como método de estudio de extensión, se incluyeron los informes de gamagrafía ósea y hepática. Todos los casos incluidos fueron diagnosticados por el servicio de patología utilizando un panel de anticuerpos contra los siguientes marcadores: citoqueratina 5/6, vimentina, EMA, calretinina, HBME-1, CEA, Ber-EP4 y TTF-1.

$\mathrm{El}$ análisis estadístico de todas las variables fue realizado con el programa SigmaStat for Windows ver. 3.5 y los gráficos con SigmaPlot for Windows ver. 11.0.

\section{Resultados}

En este trabajo mostramos la experiencia del Instituto Nacional de Enfermedades Respiratorias, que es un centro de atención de tercer nivel en la ciudad de México. Durante un período de 16 años se diagnosticaron 247 casos con MM. Observamos una tendencia al aumento en el número de casos por año (Figura 1). En la distribución general por sexos, el MM afectó principalmente a los hombres los cuales representaron un $74,7 \%$ (184), con un promedio de edad de 59 años. La mayor frecuencia de grupo se presentó 


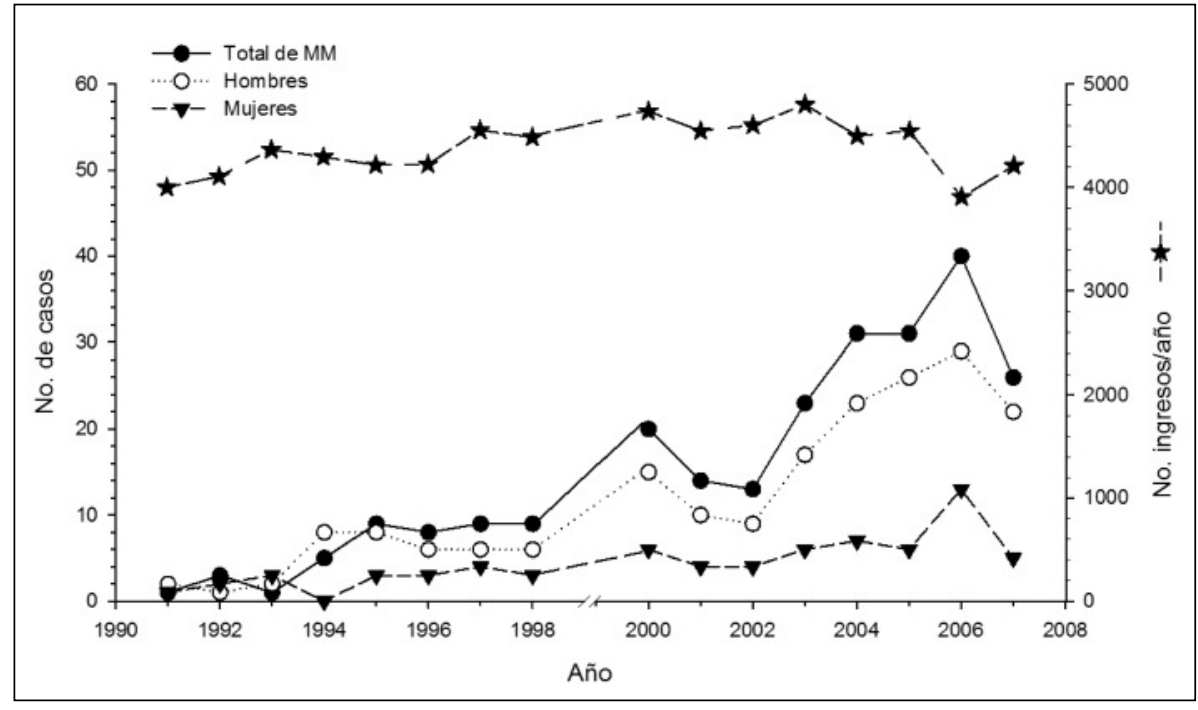

Figura 1. Frecuencia de casos de mesotelioma maligno (MM) en el INER durante el período 1991-2007. entre 51 y 60 años $(32,6 \%)$, siendo casi idéntica entre los intervalos de 41-50 años y 61-70 años. La población femenina fue un cuarto de la población $(25,5 \%)$, con una edad promedio de 58 años. Para este grupo la distribución de frecuencia fue similar en los grupos de edad de $41-50$ años y de 51-60 años, con un porcentaje de 27,0\% y $28,6 \%$ respectivamente (Tabla 1 y Figura 2). El promedio de evolución del padecimiento fue 8,8 meses, siendo mayor en mujeres que en hombres.

Los síntomas más frecuentes fueron: dolor torácico $(89 \%)$, disnea $(93 \%)$, pérdida de peso $(84 \%)$ y tos $(76 \%)$. Derrame pleural fue frecuente en este grupo de pacientes con un $87 \%$, mientras que las alteraciones radiológicas mayormente registradas fueron: engrosamiento pleural (36\%), desplazamiento del mediastino $(27 \%)$ y retracción del hemitórax (18\%). Estos resultados son similares a los notificados en la mayoría de las series de $\mathrm{MM}^{18-20}$. Dentro de los procedimientos diagnósticos empleados, en la mayoría de los pacientes el abordaje inicial incluyó toracocentesis con examen citológico del líquido pleural y de biopsia pleural. El tipo histológico más frecuente fue el epitelial seguido del sarcomatoide.

En relación con la etapa clínica, la mayoría de los pacientes presentó un IMIG IV, y varios de ellos presentaron metástasis, primordialmente en hígado y bazo. El índice de Karnofsky que presentaba la mayoría de los pacientes fue superior

Tabla 1. Características clínicas de los pacientes con Mesotelioma Maligno

\begin{tabular}{|c|c|c|c|}
\hline \multirow[t]{2}{*}{ Características generales } & \multirow[t]{2}{*}{ Total de casos } & \multicolumn{2}{|c|}{ n de casos por género } \\
\hline & & $\begin{array}{c}\text { Hombres } \\
(\%)\end{array}$ & $\begin{array}{c}\text { Mujeres } \\
(\%)\end{array}$ \\
\hline Número de pacientes & 247 & $184(74,5 \% *)$ & $63(25,5 \% *)$ \\
\hline $\begin{array}{l}\text { Edad (años) } \\
\text { Promedio } \pm \text { DS } \\
\text { Moda } \\
\text { Mediana }\end{array}$ & $\begin{array}{l}58 \pm 12 \\
50 \\
57\end{array}$ & $\begin{array}{l}59 \pm 11 \\
50 \\
57\end{array}$ & $\begin{array}{l}58 \pm 14 \\
59 \\
57\end{array}$ \\
\hline Tiempo de evolución (meses) & $8,8 \pm 14$ & $5 \pm 5$ & $15 \pm 18$ \\
\hline Fumadores & $123(49,7 \%)$ & $101(40,9 \% *)$ & $22(8,9 \%)$ \\
\hline Humo de leña & $25(10,1 \%)$ & $7(2,8 \% *)$ & $18(7,2 \%)$ \\
\hline Antecedentes oncológicos & $30(12,1 \%)$ & $20(8,0 \% *)$ & $10(4,0 \%)$ \\
\hline
\end{tabular}

*Porcentaje calculado respecto a 247 pacientes. 


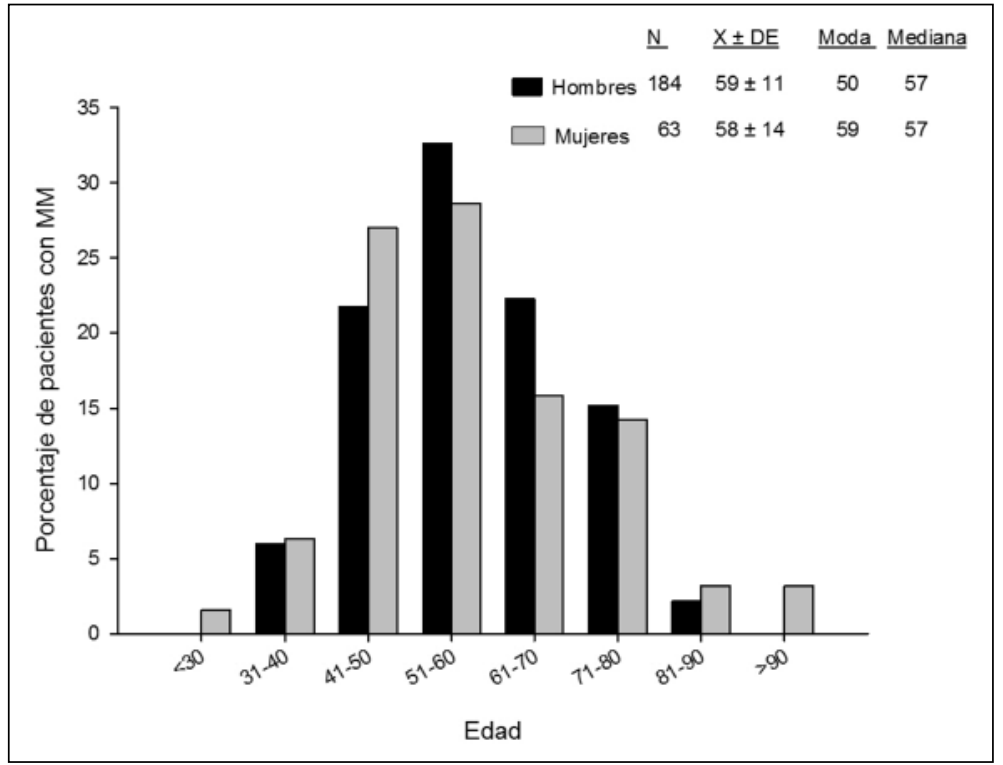

Figura 2. Distribución de pacientes con mesotelioma maligno (MM) por grupos de edad. Instituto Nacional de Enfermedades Respiratorias, México, 1991-2007.

Tabla 2. Antecedente de exposición al asbesto en pacientes con diagnóstico de Mesotelioma Maligno

\begin{tabular}{|llcc|}
\hline \multirow{3}{*}{ Hombres } & & Con exposición $(\%$ *) & Sin exposición $(\mathbf{\%}$ *) \\
& Número de casos & $57(23 \%)$ & $127(51 \%)$ \\
\multirow{2}{*}{ Mujeres } & Edad $(\overline{\mathrm{x}} \pm \mathrm{DS})$ & $61 \pm 12$ & $59 \pm 10$ \\
& Número de casos & $26(10 \%)$ & $37(15 \%)$ \\
\multirow{2}{*}{ Total } & Edad $(\overline{\mathrm{x}} \pm \mathrm{DS})$ & $52 \pm 10$ & $61 \pm 15$ \\
& Número de casos & $83(34 \%)$ & $164(66 \%)$ \\
& Edad $(\overline{\mathrm{x}} \pm \mathrm{DS})$ & $57 \pm 12$ & $59 \pm 11$ \\
\hline
\end{tabular}

*Porcentaje calculado respecto a 247 pacientes.

a 70\%, y sólo se identificó un caso con valor de $40 \%$. Los pacientes que ingresan al INER con diagnóstico de $\mathrm{MM}$, acuden en etapa clínica avanzada debido a la ausencia en etapas iniciales de sintomatología respiratoria.

Casi la mitad de los casos tuvieron tabaquismo positivo (123 casos por más de cinco años) y de ellos el porcentaje mayor estuvo representado por hombres (Tabla 1). Respecto a otro tipo de inhalantes, la exposición a humo de leña fue baja $(10 \%)$.

Históricamente se conoce la relación del asbesto y el desarrollo del mesotelioma. De los 247 pacientes estudiados solamente $34 \%$ refirieron haber tenido contacto con fibras de asbesto (57 hombres y 26 mujeres), mientras que el restante $66 \%$ de los casos no refirieron ningún tipo de exposición a asbesto. La mayoría de los casos sin antecedente de exposición al asbesto se presen- taron entre los varones (127 casos) con una edad promedio de 59 años, mientras que 37 casos sin historia de exposición a asbesto correspondió a mujeres con un promedio de edad de 61 años. (Tabla 2).

\section{Discusión}

En este estudio encontramos que el número de casos diagnosticados como MM incrementó su frecuencia anual en el INER, comenzando con un $0,03 \%$ en 1991 y elevándose hasta el $1 \%$ en el 2006 del total de patologías respiratorias. La población fue primordialmente de hombres en una relación de 2,9:1 respecto a las mujeres y con un máximo de pacientes afectados alrededor de 58 años (DS: 12 años). En un estudio previo realizado por nuestro grupo reportamos que en 
45 casos de MM, el número de hombres fue también mayor al de mujeres en una proporción de 2,7:1 y la distribución de edad fue principalmente entre los 51-60 años para ambos géneros. Estos resultados difieren muy poco de los informados en poblaciones de otras latitudes como aquella por Yates y cols ${ }^{20}$, quienes revisaron 272 casos con MM y encontraron que la edad promedio de aparición de esta neoplasia fue de 65,9 años, sin que se hayan identificado diferencias en la frecuencia del MM entre ambos sexos. En el caso de poblaciones mexicanas, Aguilar y cols ${ }^{15}$, refieren que en su estudio 793 muertes por MM, la mayor proporción también la presentaron hombres respecto a mujeres (1,5:1 respectivamente) y con una edad promedio de 60 años. Gavira y cols, reportaron 52 casos en donde la relación de géneros fue 2,5:1 y aunque en su serie la edad promedio fue menor de 50 años ${ }^{16}$.

En relación a las características clínicas y las anormalidades radiológicas, éstas fueron semejantes a las descritas en un trabajo previo ${ }^{17} \mathrm{y}$ similares a las descritas en la literatura angloamericana para el MM asociado con exposición a asbesto. Histológicamente, el mesotelioma pleural de tipo epitelial representa el $60 \%$ del total de los tipos histológicos, seguido del sarcomatoide ${ }^{6-8}$. En nuestra serie, también encontramos que el tipo histológico más frecuente observado correspondió al tipo epitelial seguido del sarcomatoide.

Respeto al tabaquismo, casi la mitad de los casos $(49,7 \%)$ refirieron ser fumadores activos, no obstante, aun no existen evidencias para pensar que exista una asociación entre el MM y el tabaquismo ${ }^{10}$. En el grupo de pacientes fumadores, sólo un $16 \%$ mencionó haber estado también expuesto a fibras de asbesto.

En la etiología del MM, el $80 \%$ de los casos presenta una historia o antecedente de exposición directa o indirecta a asbesto ${ }^{14}$. La exposición a asbesto se asocia también con la aparición de bronquitis crónica, fibrosis pulmonar y cáncer broncogénico ${ }^{21}$. Sin embargo, cabe resaltar que a la fecha no se ha podido correlacionar de manera directa la intensidad y duración de la exposición al asbesto con la aparición de enfermedad pleural y de la poca información comprobable se calcula que el riesgo para desarrollar enfermedad pleural benigna secundaria a dicha exposición es del $8 \%$ al $13 \%{ }^{6-8}$.

En nuestra serie encontramos que el porcentaje de pacientes con MM y que refirieron algún tipo de exposición a asbesto fue bajo (34\%). Aguilar y $\operatorname{cols}^{15}$, en su serie reportan que el $80 \%$ de los casos fallecidos no fueron documentados con historia de exposición al asbesto, mientras que
Gavira y $\operatorname{cols}^{16}$, sólo pudieron documentar seis casos. Cabe mencionar que toda obtención de datos a partir del expediente clínico presenta limitaciones inherentes a todo estudio retrospectivo, por lo que la ausencia de una asociación entre exposición a asbesto y el mesotelioma debe ser tomada en cuenta con algunas reservas.

En el análisis de los expedientes se describen diversas fuentes laborales de exposición a asbesto. Dos fuentes de contaminación predominan en nuestro registro: amas de casa y la contaminación ambiental. Sin embargo, estos tipos de exposición son considerados de bajo riesgo ${ }^{22,23}$, ya que cuando se trata de techos de asbesto o tinacos (depósitos domésticos de agua) el componente de asbesto-cemento conforma una matriz que encapsula a las fibras de crisotilo e impide que se desprenda al aire ambiente o al agua. Adicionalmente, tampoco se ha podido comprobar que las personas que toman agua conducida por tuberías de asbesto-cemento o los que habitan en lugares con tráfico pesado tengan mayor riesgo de enfermar. Por otra parte, las tuberías, tinacos y láminas para los techos, están fabricados con el $92 \%$ de cemento y sólo el $8 \%$ de crisotilo.

Contrariamente, la exposición se puede considerar elevada principalmente en los centros de trabajo que manejan asbesto, donde se elaboran productos con el crisotilo y en donde el trabajador se puede exponer a altas concentraciones y por tiempo prolongado. En esta serie, únicamente se encontraron cinco casos con exposición a fibras de asbesto, y sólo cuatro casos laboraron en empresas donde se utilizó crocidolita-cemento. Infortunadamente para trabajadores de asbestos en México, las fibras de anfibole (especialmente crocidolita o mezclas que contenían anfíboles) fueron ampliamente usadas hasta los $90 \mathrm{~s}$, particularmente en los trabajadores de la industria de tuberías de fibro-cemento. Es sólo a finales de los 90s que se establece la NOM-010-STPS-1999 con el propósito de mantener por debajo los límites máximos de $1 \mathrm{fibra} / \mathrm{cm}^{3}$ de aire. Debido a que el diagnóstico de MM puede presentarse de 40-45 años después de la primera exposición, varios casos más, con exposición directa a asbesto, continuarán apareciendo en los próximos años, la mayoría originados por las condiciones laborales que se tenían desde la década de los años 70's.

En términos generales, la revisión de los expedientes clínicos mostró una gran falta de datos sobre las posibles fuentes de contaminación, además de falta de información respecto al tema: i.e. manejo industrial, tipos de fibras, toxicidad, y biopersistencia asociada con la salud respiratoria. $\mathrm{Y}$ es que a pesar de la gran cantidad de estudios 
epidemiológicos de trabajadores en donde se documenta cómo los diferentes tipos de asbesto se asocian con un aumento del riesgo para la salud, en la mayoría de éstos, la exposición se produjo a través de fibras de amianto del grupo anfiboles, como son la crocidolita y amosita o mezclas de estas variedades con el crisotilo $^{24,25}$. En cambio, poco se reporta sobre trabajadores expuestos a los anfíboles o mezclas de anfíboles con crisotilo para los cuales el riesgo resulta considerablemente más bajo. De acuerdo a Hodgson y cols, el riesgo relativo para crocidolita, amosita y crisotilo es de la siguiente magnitud de 555: 100: 1. Entre las razones para estas diferencias se encuentra el fenómeno de biopersistencia por el cual anfíboles (crocidolita y amosita) son retenidos y acumulados en el pulmón por largo tiempo (años) mientras que crisotilo es eliminado rápidamente (días o semanas) ${ }^{26}$.

La incidencia de MM muestra grandes diferencias entre distintos países ${ }^{27,28}$. Estudios epidemiológicos reportan frecuencias de MM en individuos que presentan exposición alta y prolongada a asbesto que van desde el $5 \%$ hasta el $100 \%$. En los EE.UU., el 80\% de los individuos con $\mathrm{MM}$ en han sido personas expuestas a asbesto. Las razones para estas diferencias son poco claras $^{29}$. Las diferencias geográficas así como diferentes abordajes experimentales utilizados con el fin de medir la exposición pueden ser una causa de estos discrepantes resultados ${ }^{18,30}$. Así, una de las observaciones más importantes de este estudio es la dificultad de asociar la exposición a fibras de asbesto en el desarrollo del MM, por lo cual, son necesarios estudios más cuidadosos encaminados a la identificación precisa y cuantificación de fibras de asbesto. Para lo cual será necesario analizar el contenido de asbesto en el pulmón mediante microscopia óptica (cuerpos ferruginosos), o por microscopio electrónico y/o identificar la composición química de la fibra mediante técnicas como el análisis dispersivo de energía de rayos ${ }^{31}$. Infortunadamente, la mayoría de estos estudios requieren disponer de un laboratorio y de personal capacitado para preparar las muestras y efectuar el recuento de fibras o cuerpos ferruginosos. Además, cada laboratorio ha de establecer sus valores de referencia de acuerdo con la población de su entorno. Así este tipo de estudios sólo podrán realizarse en centros especializados.

\section{Conclusiones}

El estudio de los expedientes de pacientes con MM diagnosticados en el INER, muestra un incremento en el número de casos de $\mathrm{MM}$ que han sido diagnosticados en los últimos 16 años. Si bien el porcentaje de casos comparados con otras patologías respiratorias atendidas en nuestra institución aun no es muy elevado, la tendencia observada es una llamada de atención para estar alerta sobre el comportamiento epidemiológico de esta neoplasia. Las interrogantes planeadas en este estudio sugieren la necesidad de llevar a cabo estudios en un mayor número de población y en diferentes centros hospitalarios, con el fin de conocer más la biología de un tumor que a la fecha tiene pocas posibilidades de tratamiento y la sobrevida de los pacientes es muy baja. Se sugiere además que los médicos tratantes o de primer contacto, incluyan en la historia clínica de cada paciente, los antecedentes personales no patológicos la historia laboral, desde el primero hasta el último día de trabajo; los agentes a los que se expusieron y el tiempo de exposición; patología derivada del trabajo, durante su vida laboral, empresa o empresas donde laboraron, etc. Ya que de este modo sabremos con exactitud si el origen del padecimiento es del orden laboral o no y estableceremos la relación causa-efectotrabajo-daño.

\section{Agradecimientos}

Al Sr. Víctor Manuel Sánchez Nájera, por su apoyo en la recopilación estadística de casos de MM y al Sr. Juan Raúl Torres Quiles por su muy apreciable ayuda en la obtención y manejo de archivos médicos.

\section{Bibliografía}

1.- JETT J, AUBRY M. Malignant Pleural Mesothelioma. En: Clinical Respiratory Medicine: $2^{\mathrm{a}}$ edición.: Filadelfia. Mosby; 2004. Pp 735-41.

2.- MOORE A J, PARKER R J, WIGGINS J. Malignant mesothelioma. Orphanet J Rare Dis 2008; 19: 34.

3.- MCDONALD J C, MCDONALD A D. The epidemiology of mesothelioma in historical context. Eur Respir J 1996; 9: 1932-42.

4.- BIANCHI C, GIARELLI L, GRANDI G, BROLLO A, RAMANI L, ZURCH C. Latency periods in asbestos related mesothelioma of the pleura. Eur J Cancer Prev 1997; 6: 162-6.

5.- ZELLOS L, CHRISTIANI D C. Epidemiology, biologic behavior, and natural history of mesothelioma. Thorac Surg Clin 2004; 14: 469-77.

6.- ALBEDA S M, STERMAN D H, LITZKY L A. Malignant mesothelioma and other primary pleural tumors. En: Fishman's pulmonary diseases and disorders. 
Editado por Fishman AP, Elias JA, Fishman JA, Grippi MA, Kaiser LR, Senior RM. $3^{\text {a }}$ Edición. Nueva York. McGraw-Hill; 1998; Pp. 1453-66.

7.- STEWART D J, EDWARDS J G, SMYTHE W R, WALLER D A, O'BYRNE K J. Malignant pleural mesothelioma - an update. Int J Occup Environ Health 2004; 10: 26-39.

8.- HUGHES R S. Malignant pleural mesothelioma. Am J Med Sci 2005; 329: 29-44.

9.- ROBINSON B W, LAKE R A. Advances in malignant mesothelioma. N Engl J Med 2005; 353: 1591-603.

10.- YANG H, TESTA J R, CARBONE M. Mesothelioma epidemiology, carcinogenesis, and pathogenesis. Curr Treat Options Oncol 2008; 9: 147-57.

11.- WEILL H, HUGHES J M, CHURG A M. Changing trends in US mesothelioma incidence. Occup Environ Med 2004; 61: 438-41.

12.- PETO J, HODGSON J T, MATTHEWS F E, JONES $\mathrm{J} R$. Continuing increase in mesothelioma mortality in Britain. Lancet 1995; 345: 535-9.

13.- HODGSON J T, MCELVENNY D M, DARNTON A J, PRICE M J, PETO J. The expected burden of mesothelioma mortality in Great Britain from 2002 to 2050 . Br J Cancer 2005; 92: 587-93.

14.- NISHIKAWA K, TAKAHASHI K, KARJALAINEN A, WEN CP, FURUYA S, HOSHUYAMA T, TODOROKI $\mathrm{M}$, et al. Recent mortality from pleural mesothelioma, historical patterns of asbestos use, and adoption of bans: a global assessment. Environ Health Perspect 2008; 116 1675-80.

15.- AGUILAR-MADRID G, JUÁREZ-PÉREZ C A, MARKOWITZ S, HERNÁNDEZ-ÁVILA M, SÁNCHEZ-ROMÁN FR, VÁZQUEZ GRAMEIX J H. Globalization and the transfer of hazardous industry: asbestos in Mexico, 1979-2000. Int J Occup Environ Health 2003; 9: 272-9.

16.- GAVIRA-SÁNCHEZ L, MARÍN-LÓPEZ M, URTIAGA-DOMÍNGUEZ M. El asbesto y la salud en la ciudad de México: un caso de transferencia del riesgo y del consumo. Salud Problema 1990; 19: 31-45.

17.- GARCÍA-LÓPEZ M DEL P, BARRERA-RODRÍGUEZ R. Mesotelioma maligno: descripción clínica y radiológica de 45 casos con y sin exposición a asbesto. Salud pública Méx 2000; 42: 511-9.

18.- YANG H, TESTA J R, CARBONE M. Mesothelioma epidemiology, carcinogenesis, and pathogenesis. Curr
Treat Options Oncol 2008; 9: 147-57.

19.- BERRY M. Mesothelioma incidence and community asbestos exposure. Environ Res 1997; 75: 34-40.

20.- YATES D H, CORRIN B, STIDOLPH P N, BROWNE K. Malignant mesothelioma in south east England: clinicopathological experience of 272 cases. Thorax 1997 52: 507-12.

21.- UPADHYAY D, KAMP D W. Asbestos-induced pulmonary toxicity: role of DNA damage and apoptosis. Exp Biol Med (Maywood) 2003; 228: 650-9.

22.- LADOU J. Current ocupational and enviromental medicine. $4^{\circ}$ Edición. Nueva York. McGraw Hill; 2006.

23.- ROGGLI V L, SHARMA A, BUTNOR K J, SPORN $\mathrm{T}$, VOLLMER R T. Malignant mesothelioma and occupational exposure to asbestos: a clinicopathological correlation of 1445 cases. Ultrastruct Pathol 2002; 26 : 55-65.

24.- BERNSTEIN D, ROGERS R, SMITH P. The biopersistence of Canadian chrysotile asbestos following inhalation: final results through 1 year after cessation of exposure. Inhalation Toxicol 2005; 17: 1-14.

25.- BERNSTEIN D M, HOSKINS J A. The health effects of chrysotile: current perspective based upon recent data Regul Toxicol Pharmacol 2006; 45: 252-64.

26.- HODGSON J T, DARNTON A. The quantitative risks of mesothelioma and lung cancer in relation to asbestos exposure. Ann Occup Hyg 2000; 44: 565-601.

27.- BIANCHI C, BIANCHI T. Malignant mesothelioma: global incidence and relationship with asbestos. Ind Health 2007; 45: 379-87.

28.- BECKLAKE M R, BAGATIN E, NEDER J A. Asbestos-related diseases of the lungs and pleura: uses, trends and management over the last century. Int $\mathrm{J}$ Tuberc Lung Dis 2007; 11: 356-69.

29.- CARBONE M, RDZANEK M A. Pathogenesis of malignant mesothelioma. Clin Lung Cancer 2004; 5 (Suppl 2): S46-S50.

30.- WEINER S J, NERAGI-MIANDOAB S. Pathogenesis of malignant pleural mesothelioma and the role of environmental and genetic factors. J Cancer Res Clin Oncol 2009; 135: 15-27.

31.- DE VUYST P, KARJALAINEN A, DUMORTIER P, PAIRON JC, MONSÓ E, BROCHARD P, et al. Guidelines for mineral fibre analyses in biological samples: report of the ERS Working Group. Eur Respir J 1998; 11: $1416-26$

Correspondencia a:

Dr. Raúl Barrera Rodríguez

Depto. Bioquímica y Medicina Ambiental

Instituto Nacional de Enfermedades Respiratorias

Clza. Tlalpan, 4502, Col Sec. XVI., México, D.F.,

14080, México.

Fax: 52-5554871772

E-mail: barrerarr@hotmail.com raulbarrera@iner.gob.mx 